\title{
Immobilized cyclopentadiene in the Diels Alder/retro-Diels Alder concept
}

\author{
René G. Gieling and Antonius J. H. Klunder* \\ Department of Organic Chemistry, NSRIM, University of Nijmegen, \\ Toernooiveld 1, 6525 ED Nijmegen, The Netherlands \\ E-mail:ajhk@sci.kun.nl
}

Dedicated to Professor Binne Zwanenburg on the occasion of his $70^{\text {th }}$ birthday

(received 28 Oct 03; accepted 11 Dec 03; published on the web 19 Dec 03)

\begin{abstract}
Immobilized cyclopentadiene on polystyrene has been synthesized and studied in $[4 \pi+2 \pi]$ cycloadditions with a variety of dienophiles. Reasonable to excellent conversions were obtained. The cyclopentadiene Diels-Alder adducts turned out to be generally too stable to allow effective cycloreversion both under dynamic (under vacuum) and static (in a solvent) thermal conditions. A detailed study of the immobilized cyclopentadiene benzoquinone adduct showed a remarkable inreactivity of the enone double bond showing the limitations of the solid phase approach in the Diels-Alder/Retro-Diels-Alder synthetic strategy.
\end{abstract}

Keywords: Merrifield resin, Wang resin, tetracyanoethylene

\section{Introduction}

The Diels Alder/retro-Diels Alder concept is a powerful synthetic strategy in stereo- and enantioselective routes to functionalized cycloalkenes. ${ }^{1,2}$ We have extensively studied this concept for the endo-tricyclo[5.2.1.0 $0^{2,6}$ decadienone system $\mathbf{1}^{2 \mathrm{~d}, 3}$ (Scheme 1). This tricyclic enone is in essence the Diels Alder adduct of cyclopentadiene and the elusive cyclopentadienone 2. The annulated cyclopentenone moiety in $\mathbf{1}$ can be chemically transformed, e.g. by conjugated additions to afford functionalized tricyclic decadienones $\mathbf{3}$. The reversibility of the Diels Alder reaction now allows regeneration of an enone moiety affording functionalized cyclopentenones 4. This cycloreversion can be accomplished thermally, either in solution (static thermolysis) or in the gas phase (dynamic thermolysis). For gas phase reactions the Flash Vacuum Thermolysis (FVT) technique has been particularly successful for those substrates that have a sufficiently high volatility. ${ }^{4,5}$ Since the formation of cyclopentenones $\mathbf{4}$ from cyclopentadienone $\mathbf{2}$ by direct conjugate additions is not possible due to the instability of cyclopentadienone 2 above $-100^{\circ} \mathrm{C}$, 
the tricyclodecadienone system $\mathbf{1}$ in fact constitutes a synthetic equivalent of cyclopentadienone 2.

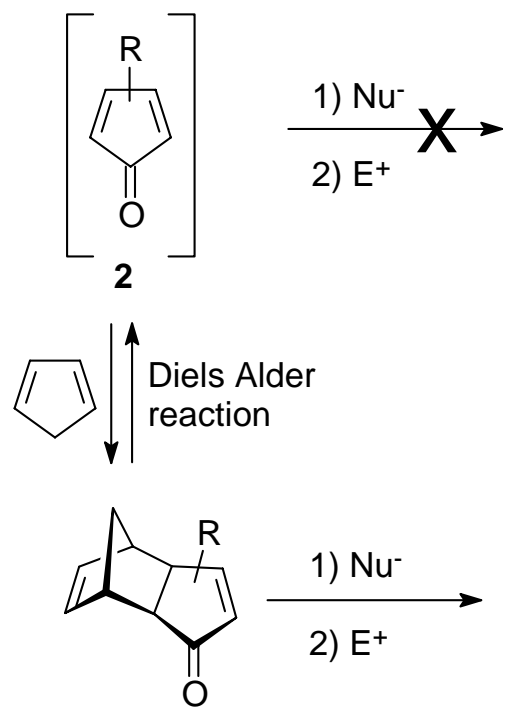

1

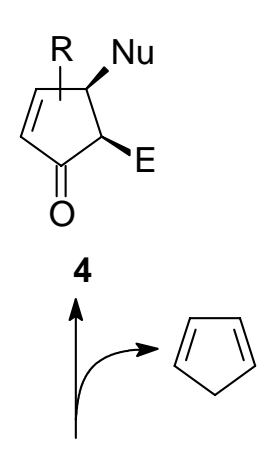<smiles>[R]C1C(F)C([NH])C(=O)C2C3C=CC(C3)C12</smiles>

3

\section{Scheme 1}

In principle, the Diels Alder/retro-Diels Alder concept can be translated into a process that is carried out on solid support. Such a solid-phase process could possibly lead to a combinatorial synthesis of cycloalkenes. This approach would feature the immobilized component $\mathbf{5}$ as the protecting cyclic diene in the initial Diels Alder reaction affording the resin-bound adduct $\mathbf{7}$ when reacted with dienophile 6 (Scheme 2). Chemical modification of the immobilized tricyclic system $\mathbf{7}$ to $\mathbf{8}$ is followed by thermal cycloreversion cleaving the modified dienophile $\mathbf{9}$ from the immobilized cyclic diene 5 that in principle could be reused.
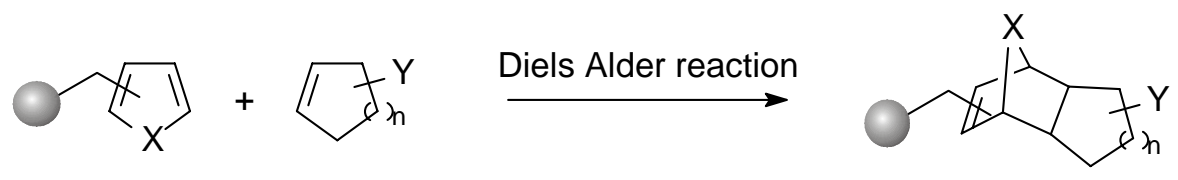

$$
\begin{aligned}
& \mathbf{5 a} X=\mathrm{CH}_{2} \quad \mathbf{6} \\
& \mathbf{5 b} \mathrm{b}=\mathrm{O}
\end{aligned}
$$<smiles>[Y]1C=CC=CC1Cc1ccccc1</smiles>

5

9

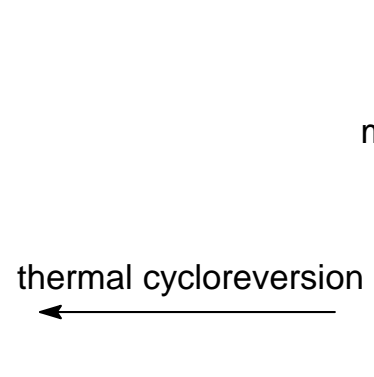

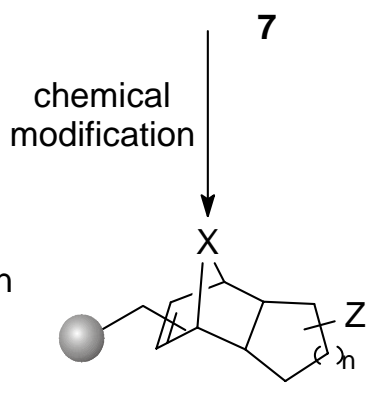

8

\section{Scheme 2}


In this paper, the synthesis and reactivity of immobilized cyclopentadiene 5a and some selected adducts, is reported. Only a few papers on immobilized cyclopentadiene have been reported hitherto. A detailed study of the general application of immobilized cyclopentadiene in the Diels Alder/retro-Diels Alder synthetic strategy has not yet been undertaken. In 1985, Mirua et al. ${ }^{6}$ disclosed a first example of polymer-supported cyclopentadiene 5a. Rotello et al. ${ }^{7}$ reported the Diels Alder reaction between buckminsterfullerene $\mathrm{C}_{60}$ and cyclopentadiene linked to the Merrifield resin. Recently, Wanner and Koomen ${ }^{8}$ showed the ability of immobilized cyclopentadiene 5a to react with the vulnerable nitroso functionality of nitrosoadenosine triacetate. Rotello et al. ${ }^{9}$ also investigated cyclopentadiene immobilized on silica gel as an extension of their studies on the purification of buckminsterfullerenes.

\section{Results and Discussion}

Rotello et al. ${ }^{7}$ immobilized cyclopentadiene by stirring a solution of sodium cyclopentadienylide in THF with the Merrifield resin 10 at $-20^{\circ} \mathrm{C}$. A loading of $52 \%$ was thus accomplished, i.e. $52 \%$ of available chloride of Merrifield resin $\mathbf{1 0}$ had been substituted by cyclopentadiene. A significant improvement of Rotello's procedure was achieved by simply increasing the reaction temperature from $-20^{\circ} \mathrm{C}$ to $45^{\circ} \mathrm{C}$ and using $\mathrm{THF}$ as the solvent. A loading of $70 \%$ was now achieved (Table 1, entry 1). The higher temperatures did not adversely affect the stability of the sodium cyclopentadienylide solution, as long as the reaction was performed under a strict inert atmosphere. ${ }^{10}$ It should be noted that the Merrifield resin used here is different from the one applied by Rotello, regarding the degree of cross-linking and the number of available sites (see experimental part). The use of lithium as the cation did not lead to better results (Table 1, entries $2,4)$. This observation is in agreement with the loading of $43 \%$ reported by Wanner and Koomen, ${ }^{8}$ when they used lithium cyclopentadienide in DMF.

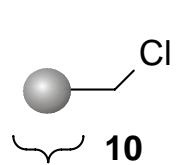

styrene/ divinylbenzene copolymer

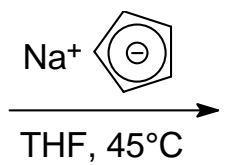

$\mathrm{THF}, 45^{\circ} \mathrm{C}$

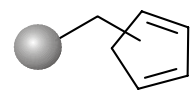

$5 a$

\section{Scheme 3}


Table 1. Linking of Cyclopentadiene to the Merrifield resin

\begin{tabular}{lllll}
\hline Entry & Solvent & Cation & Loading $^{\mathrm{a}}(\mathrm{mmole} / \mathrm{g})$ & Substitution $^{\mathrm{b}}(\%)$ \\
\hline 1 & THF & $\mathrm{Na}^{+}$ & 1.19 & 70 \\
2 & THF & $\mathrm{Li}^{+}$ & 0.69 & 41 \\
3 & Toluene & $\mathrm{Na}^{+}$ & 1.09 & 64 \\
4 & Toluene & $\mathrm{Li}^{+}$ & 0.08 & 4 \\
\hline
\end{tabular}

${ }^{\mathrm{a}}$ Cyclopentadiene (in mmole/g functionalised resin) that reacted with tetracyanoethylene.

${ }^{\mathrm{b}}$ Percentage of chloride in Merrifield resin that is substituted by cyclopentadiene.

The immobilization reaction was monitored with infrared spectroscopy. The Merrifield resin gave a sharp absorption at $1265 \mathrm{~cm}^{-1}$ due to $\mathrm{C}$-Cl-stretching. This absorption decreased in intensity when the reaction proceeded.

The loading was determined by two methods. The first method constitutes the quantitative determination of the chloride that is released in the substitution of the Merrifield resin with the cyclopentadienyl anion. After the immobilization reaction, the resin was washed with water. The combined aqueous washings contained the chloride anions substituted by cyclopentadiene. The quantity of these chloride anions was determined by titration in triplo using the Vollhard titration. ${ }^{11}$ Assuming that this quantity of chloride corresponds to an equimolar amount of reacted cyclopentadiene, the loading can now be easily calculated. The second method is based upon a report by Bing Yan and co-workers ${ }^{12}$ who showed that elemental analysis of resins provides reliable information on the presence of certain elements, e.g. nitrogen. For this purpose, the immobilized cyclopentadiene 5a was reacted with tetracyanoethylene (Scheme 4). Elemental analysis of the resulting immobilized adduct $\mathbf{1 1 b}$ provided the nitrogen content of the resin, thus giving the amount of tetracyanoethylene that had reacted. As it is assumed that tetracyanoethylene reacts quantitatively with cyclopentadiene, the cyclopentadiene loading can now easily be calculated.

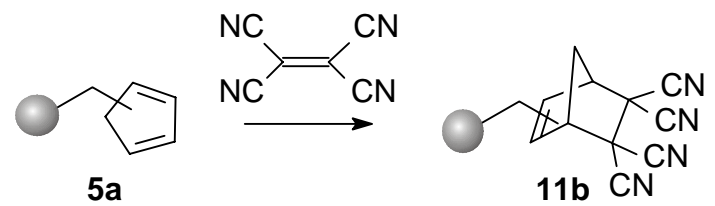

\section{Scheme 4}

In a typical immobilization reaction, the titration method showed that 1.19 mmole of available chloride per gram of Merrifield resin was substituted by cyclopentadiene, resulting in a loading of 1.15 mmole cyclopentadiene per gram of resin 5a. Elemental analysis of the tetracyanoethylene adduct showed that 1.25 mmole cyclopentadiene per gram of $\mathbf{5 a}$ had reacted. These results show that the immobilized cyclopentadiene had indeed reacted quantitatively with tetracyanoethylene. In conclusion, elemental analysis of the tetracyanoethylene adduct $\mathbf{1 1 b}$ gives a reliable estimation of the loading of cyclopentadiene on a polystyrene resin and is a good alternative for the chloride titration method. 
Two examples of a Diels Alder reaction applying immobilized cyclopentadiene on a polystyrene-based resin were known at the time this research started. Miura and co-workers ${ }^{6}$ described the cycloaddition of immobilized cyclopentadiene with maleic anhydride in nitrobenzene at $180^{\circ} \mathrm{C}$. Rotello et al. ${ }^{7,9}$ disclosed the cycloaddition with a mixture of $\mathrm{C}_{60}$ and $\mathrm{C}_{70}$ buckminsterfullerenes.

Besides the successful cycloaddition of immobilized cyclopentadiene $\mathbf{5 a}$ with tetracyanoethylene as described above, the Diels-Alder reactivity of $\mathbf{5 a}$ was studied for a variety of dienophiles (Scheme 5). All dienophiles used were commercially available except for $\mathrm{N}$ phenethylmaleimide ${ }^{13}$ and diethyl maleate.

The Diels Alder reactions were generally carried out overnight in DMF at room temperature. Only diethyl maleate required an elevated temperature to achieve acceptable conversions. This in accordance with the relatively low reactivity of this dienophile towards cyclopentadiene in solution. The formation of the Diels Alder adducts was confirmed by IR analysis. The benzoquinone adduct 11c, for example, showed a $\mathrm{C}=\mathrm{O}$ absorption at $1670 \mathrm{~cm}^{-1}$. The maleic anhydride adduct 11a showed typical anhydride $\mathrm{C}=\mathrm{O}$ absorptions at 1860 and $1773 \mathrm{~cm}^{-1}$ and $\mathrm{C}$ $\mathrm{O}$ stretches at $1221 \mathrm{~cm}^{-1}$, whereas the diethyl maleate adduct 11d had a characteristic ester absorption at $1728 \mathrm{~cm}^{-1}$. Weak CN-absorptions were observed at $2200 \mathrm{~cm}^{-1}$ in the IR-spectra of the tetracyanoethylene adduct 11b. The yields were determined by measuring the weight increase of the resin or more accurately by elemental analysis when nitrogen was present in the dienophiles and hence in the polymer-supported adducts. The results listed in Table 2 show that all cycloaddition reactions can be performed with high efficiency.

Table 2. Diels Alder reactions with cyclopentadiene immobilized on Merrifield resin

\begin{tabular}{|c|c|c|c|c|c|}
\hline Entry & Diels Alder adduct of & $\begin{array}{l}\text { Starting } \\
\text { resin } \\
(\mathrm{mmole} / \mathrm{g})\end{array}$ & $\begin{array}{l}\text { Cyclopentadiene } \\
\text { Reacted }^{\mathrm{b}} \\
\text { (mmole/g) }^{\text {(mmole }}\end{array}$ & $\begin{array}{l}\text { Loading }^{\mathrm{c}} \\
\text { (mmole/g) }\end{array}$ & $\begin{array}{l}\text { Conversion } \\
\text { (\%) }\end{array}$ \\
\hline 1 & Maleic anhydride 11a & 1.19 & 0.80 & 0.74 & 67 \\
\hline 2 & Tetracyanoethylene 11b & 1.19 & 1.25 & 1.08 & 100 \\
\hline 3 & Benzoquinone 11c & 0.91 & 0.77 & 0.70 & 85 \\
\hline 4 & Diethyl maleate $\mathbf{1 1 d}^{\mathrm{d}}$ & 0.91 & 0.39 & 0.37 & 43 \\
\hline 5 & Acrolein 11e & 1.19 & 0.94 & 0.89 & 79 \\
\hline 6 & Maleimide 11f & 0.91 & 0.64 & 0.61 & 70 \\
\hline 7 & 2-Chlorobenzoquinone $\mathbf{1 1 g}$ & 1.19 & 1.04 & 0.90 & 87 \\
\hline 8 & $\mathrm{~N}$-phenethylmaleimide $\mathbf{1 1 h}$ & 1.19 & 0.95 & 0.80 & 80 \\
\hline
\end{tabular}

${ }^{\mathrm{a}}$ Loading of cyclopentadiene on starting resin.

${ }^{\text {b }}$ Calculated by weight increase, except for entries 2, 5, 8 (calculated by elemental analysis).

${ }^{\mathrm{c}}$ Loading of Diels Alder adduct, expressed as mmole/g modified resin.

${ }^{\mathrm{d}}$ Reaction performed at $80^{\circ} \mathrm{C}$. 


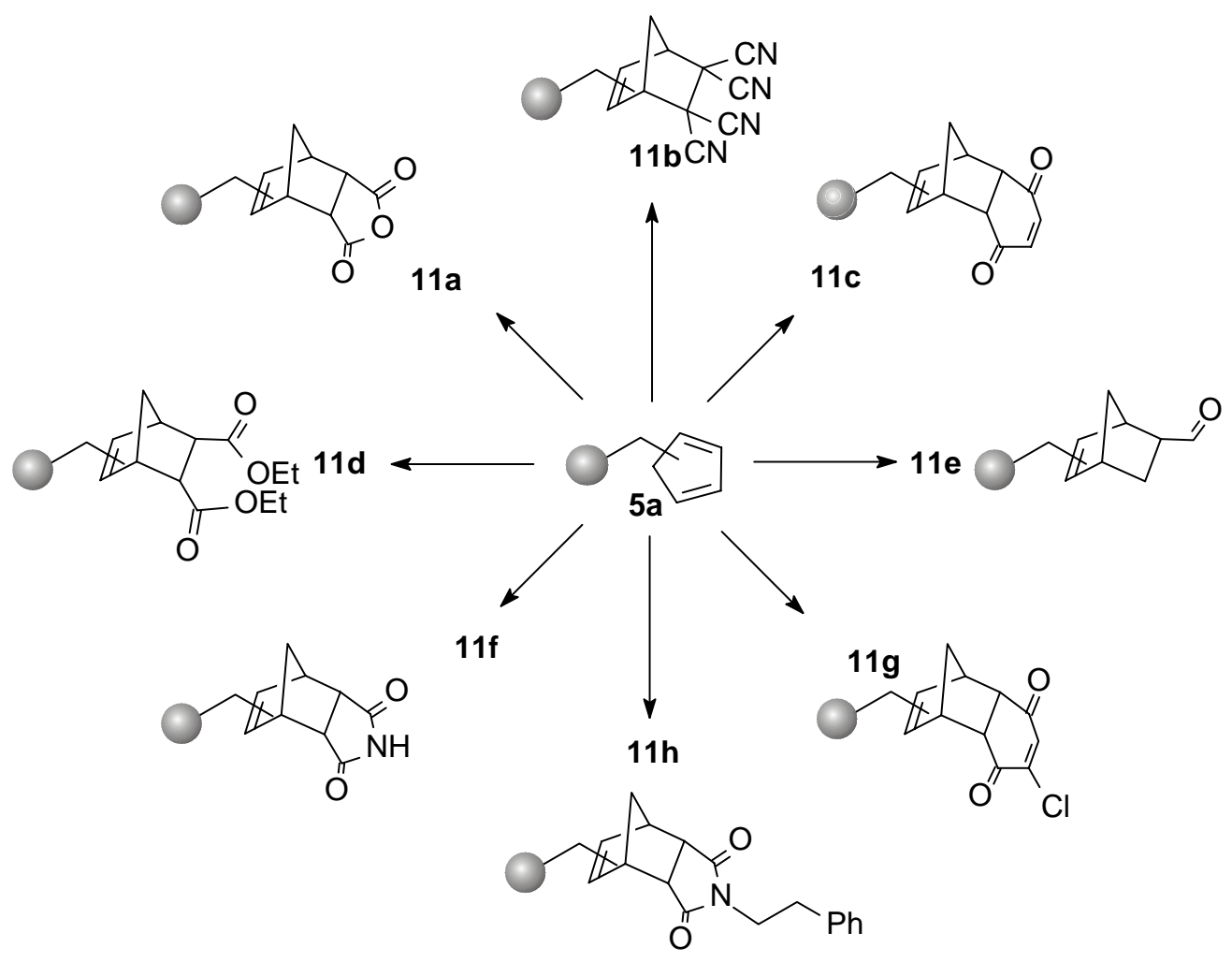

\section{Scheme 5}

In solution, monosubstituted cyclopentadienes rapidly isomerize at room temperature through a 1,5 hydride shift (Scheme 6). This isomerisation is catalyzed by alkali cyclopentadienylide. ${ }^{14,15}$ As a result, the reaction of an alkyl halide with sodium cyclopentadienylide always leads to a mixture of three possible alkylcyclopentadiene isomers 12a-c. ${ }^{15,16,17}$ In Diels Alder cyclizations, alkylcyclopentadienes generally afford mixtures of the corresponding cycloadducts 13a-c, in which the alkylgroup may be attached at a maximum of three different positions (Scheme 6).<smiles>[R1]C1C=CC=C1</smiles>

$12 a$

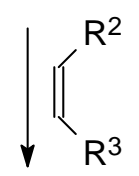<smiles>[R]C1C([R])C2C=CC1C2[R]</smiles>

$13 a$

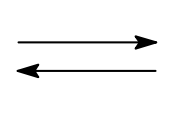

12

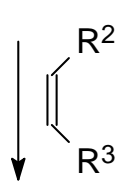<smiles>[R]C1C2C=CC(C2)C([R])C1[R]</smiles>

$13 b$

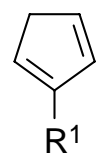

12c

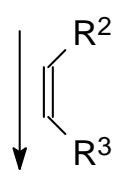<smiles>[R]C1=CC2CC1C([R])C2[R]</smiles>

13c

\section{Scheme 6}


Similarly, immobilization of cyclopentadiene on a resin most likely also gives rise to three isomerizing structures. As a consequence, the produced immobilized cycloadducts $\mathbf{1 2}$ may be linked at three different positions to the polystyrene-based resin. It is likely that the mode of linkage has a direct impact on both the subsequent chemical transformations carried out on these polycyclic structures and the eventual cycloreversion process. Therefore, it is desirable to know whether there is any regioselectivity in the $[4 \pi+2 \pi]$-cycloadditions of immobilized cyclopentadiene. Hence, attempts were made to analyze the composition of a possible isomeric mixture 11c (Figure 1), obtained from the reaction of immobilized cyclopentadiene 5a and benzoquinone.

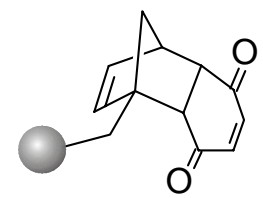

(I)

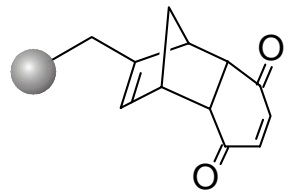

(II)

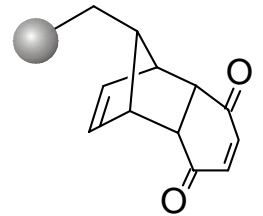

(III)

Figure 1. The three different isomers of immobilized cycloadduct 11c.

A realistic structure determination is only possible after cleavage of the complete cycloadducts from the resin. In order to cleave the entire tricyclic structures from the resin, a cleavable linker must be introduced between the polymeric backbone and cyclopentadiene. The Wang resin was chosen, because it has an additional cleavable methoxyphenyl linker, which is lacking in the Merrifield resin. Cleavage from the Wang resin under acidic conditions normally occurs by breaking an ester bond. However, if the benzoquinone adduct of cyclopentadiene is immobilized on the Wang resin as in structure 17, only an ether bond is present for cleavage. This bond can readily be broken by using trifluoroacetic acid or boron tribromide. ${ }^{18}$ The resulting mixture of isomers 18 can then be analyzed, e.g. by GC/MS.

In order to immobilize cyclopentadiene on the Wang resin 14, the hydroxyl groups of this resin first need to be substituted by chloride. This was accomplished by treatment of $\mathbf{1 4}$ with dichlorotriphenylphosphorane in dichloromethane in the presence of imidazole, affording resin 15. ${ }^{19}$ Cyclopentadiene was now easily immobilized on the Wang resin using the same conditions as those that are optimal for linking to the Merrifield resin (Scheme 7). Subsequent cycloaddition of benzoquinone and the obtained resin $\mathbf{1 6}$ gave the Wang-immobilized adduct $\mathbf{1 7}$ with a loading of 0.75 mmole per gram of resin. 


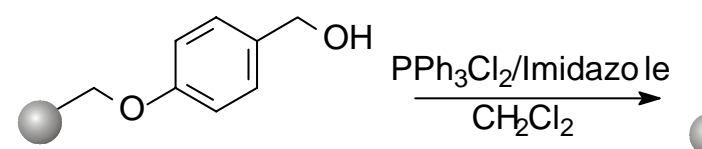

14

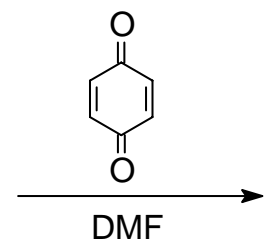

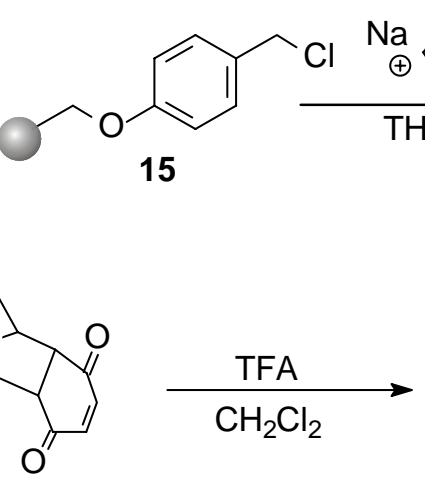

17

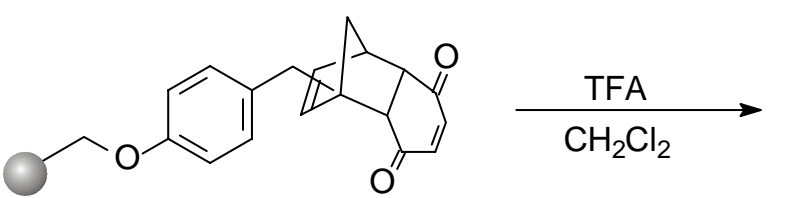

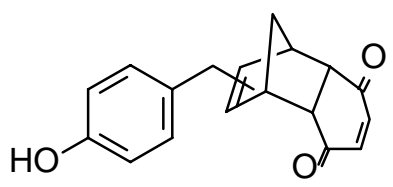

18

\section{Scheme 7}

Immobilized adduct $\mathbf{1 7}$ was then treated with trifluoroacetic acid in dichloromethane, followed by thorough washing of the resin. The combined washings were concentrated, affording a complex mixture. The mixture was analysed with GC-MS in order to establish whether isomers of tricyclic structure $\mathbf{1 8}$ were present. The gas chromatogram showed three peaks in a ratio of 2:1:1. The mass spectra associated with these peaks contained parent ions of $280 \mathrm{~m} / \mathrm{z}$ and similar fragmentation patterns, confirming the cleavage of three isomers of tricyclic structure $\mathbf{1 8 .}$

The abundance of one isomer over the other two isomers indicates that attachment of cyclopentadiene to the resin at one position is preferred. This is in accordance with the observation that the 5-substituted alkylcyclopentadiene 12a is the kinetically preferred product in solution. ${ }^{14,15}$ The resulting immobilized Diels Alder adduct is most likely the bridgehead substituted tricyclic structure III (Figure 1). However, this cannot be confirmed unambiguously by mass spectroscopy alone. In conclusion, some selectivity in the formation of the immobilized cyclopentadiene adducts is observed. Apparently, there is no effect of the polystyrene backbone on the isomeric ratio.

In order to study the efficiency of the cycloreversion of the immobilized cycloadducts 11a,c,d,g and $\mathbf{h}$, two methods were applied, viz. thermolysis of the resin beads under vacuo and trapping of the volatile products at $-78^{\circ} \mathrm{C}$ (dynamic procedure) and heating the resin beads in a high boiling solvent (static procedure). In the first approach the immobilized cycloadducts were heated for three hours at a pressure of 0.05 mbar and applying temperatures varying between 150 to $250^{\circ} \mathrm{C}$. Higher temperatures were not applied as considerable decomposition (blackening) of the resin took place. At $150^{\circ} \mathrm{C}$, cycloreversion was only observed for adduct 11c, affording benzoquinone in small amounts. The other adducts did not undergo cycloreversion until a temperature of $200^{\circ} \mathrm{C}$ was attained. The yield of cleavage products at this temperature was generally rather low as shown in Table 3, except for benzoquinone. Maleic anhydride could also be obtained in a good yield, albeit only at $250^{\circ} \mathrm{C}$. 
Table 3. Results of the cycloreversion reactions under dynamic conditions

\begin{tabular}{lllll}
\hline \multirow{2}{*}{ Entry } & \multirow{2}{*}{ Immobilized adduct } & \multicolumn{3}{l}{ Yield of Cleavage Product (mmole/g) } \\
& & $150^{\circ} \mathrm{C}$ & $200^{\circ} \mathrm{C}$ & $250^{\circ} \mathrm{C}$ \\
\hline 1 & $\mathbf{1 1 a}$ & 0 & $0.20(29 \%)$ & $0.66(89 \%)$ \\
2 & $\mathbf{1 1 c}$ & $0.03(19 \%)$ & $0.65(93 \%)$ & $\mathrm{a}$ \\
3 & $\mathbf{1 1 d}$ & 0 & $0.16(43 \%)$ & $\mathrm{a}$ \\
4 & $\mathbf{1 1 g}$ & 0 & $0.22(24 \%)$ & $\mathrm{a}$ \\
5 & $\mathbf{1 1 h}$ & 0 & $0.02(3 \%)$ & $0.08(10 \%)$ \\
\hline
\end{tabular}

${ }^{\mathrm{a}}$ not determined.

This dynamic thermolysis approach may have the disadvantage that less volatile dienophiles produced may not be completely released from the resin and therefore lower the observed yield. In order to quantify this problem, the residual resin was swollen in dichloromethane, stirred and filtered. Careful analysis of this filtrate showed the absence of any cycloreversion product indicating that the rational given above does not explain the observed low yields. Finally, the same heating protocol was repeated, but this did not result in an additional amount of dienophile.

The immobilized benzoquinone adduct 11c was also subjected to the static thermolysis protocol. Thus, adduct 11c was heated at reflux in a high-boiling solvent, such as dimethylformamide or $o$-dichlorobenzene. After filtration of the resin, the filtrate was analyzed with GC and TLC to establish whether any cycloreversion product had been cleaved off from the resin. The results listed in Table 4 reveal that no cycloreversion could be achieved at all, using this approach. Apparently, the temperature needed for cycloreversion of solid phase cyclopentadiene adducts 11c under static thermal conditions is considerably higher than required under dynamic thermal conditions. Because of these negative results with 11c, the other cycloadducts $\mathbf{1 1}$ were not studied under these conditions.

Table 4. Attempted cycloreversion of 11c in a solvent under static thermal conditions

\begin{tabular}{lllll}
\hline Entry & Solvent & Temperature $\left({ }^{\circ} \mathrm{C}\right)$ & Reaction time & Cycloreversion \\
\hline 1 & DMF & 150 & $3 \mathrm{~h}$ & $\mathrm{X}$ \\
2 & DMF & 150 & $17 \mathrm{~h}$ & $\mathrm{X}$ \\
3 & DMF & 150 & $5 \mathrm{~min}^{\mathrm{a}}$ & $\mathrm{X}$ \\
4 & $o$-dichlorobenzene & 240 & $17 \mathrm{~h}$ & $\mathrm{X}$ \\
\hline
\end{tabular}

${ }^{a}$ microwave, 500W.

Recapitulating, the temperature required for cycloreversion of cycloadducts $\mathbf{1 1}$ is generally too high and highly dependant on the nature of the dienophile to be released. Besides, the only successful cycloreversion reactions were performed under dynamic thermolysis conditions. 
However, with respect to a combinatorial extension of the Diels-Alder/retro-Diels-Alder concept, cycloreversion under static thermolysis conditions, i.e. using a solvent, would be preferable. Cycloreversion of immobilized cyclopentadiene adducts by thermolysis is possible, but the results are not satisfactory for a combinatorial approach.

The benzoquinone adduct 11c was now selected for a study on conceivable chemical transformations. Such a modified adduct would produce a new cyclohexene compound when subjected to thermolysis. If successful this would substantiate the viability of the Diels Alder/retro-Diels Alder concept on solid phase as depicted in Scheme 2.

The immobilized benzoquinone adduct 11c was subjected to the alkaline epoxidation reaction (Scheme 8). Although this reaction proceeds readily in solution, ${ }^{20}$ no epoxidation took place at all as was shown by infrared spectroscopy. In addition, thermolysis of the resulting resin afforded benzoquinone as the only cycloreversion product. A possible explanation might be the poor swelling capacity of the solvent mixture used, viz. acetone and water. The active sites on the resin may not be accessible for the basic hydroperoxide, blocking the epoxidation. However, other epoxidation methods using more suitable solvents (Table 5, entries 2,3), including one method especially designed for solid-phase synthesis (Table 5 , entry 4 ), ${ }^{21}$ did not alter the result. The desired epoxide 19 was not formed. Hence, it can be concluded that other factors than a poor swelling capacity prevent the epoxidation of immobilized adduct 11c.

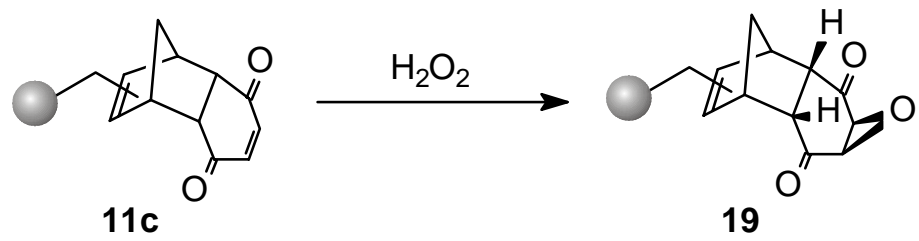

\section{Scheme 8}

Table 5. Methods tried for the epoxidation of immobilized benzoquinone adduct 11c

\begin{tabular}{llll}
\hline Entry & Solvent & Base & Oxidizing agent \\
\hline 1 & Acetone/water & $\mathrm{Na}_{2} \mathrm{CO}_{3}$ & $\mathrm{H}_{2} \mathrm{O}_{2}$ \\
2 & Dichloromethane/methanol & aq $\mathrm{NaOH}$ & $\mathrm{H}_{2} \mathrm{O}_{2}$ \\
3 & Dichloromethane & aq $\mathrm{Na}_{2} \mathrm{CO}_{3}$ & $\mathrm{H}_{2} \mathrm{O}_{2}$ \\
4 & Dichloromethane/trichloroacetonitrile & $\mathrm{K}_{2} \mathrm{HPO}_{4}$ & $\mathrm{H}_{2} \mathrm{O}_{2}$ \\
\hline
\end{tabular}

Other reactions that work well for non-immobilized tricyclic cyclopentadiene benzoquinone adducts, viz. aromatisation of the quinone system or a [4+2]-Diels Alder reaction with dimethylbutadiene were also tried with immobilized adduct 11c. However, only starting material was recovered. These negative results reveal that the enone moiety in immobilized adduct 11c is unexpectedly unreactive. 
Theoretically, it is possible that the double bond has undergone a second Diels Alder reaction with another immobilized cyclopentadiene moiety, to give the cross-linked polystyrene resin $\mathbf{2 0}$, as shown in Figure 2. The high degree of cross-linking in resin $\mathbf{2 0}$ should be obvious from deteriorated swelling properties. However, when immobilized adduct 11c was suspended in dichloromethane, no reduced swelling compared to the Merrifield resin was observed.

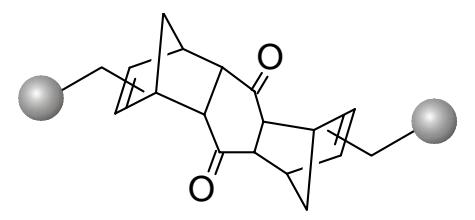

20

Figure 2. Cross-linking in immobilized adduct 11c.

Additional experiments had to be performed to obtain more information on the possible existence of cross-linked structure 20. Both the bis-cyclopentadiene benzoquinone adduct 22 and its immobilized counterpart 23 were synthesized for comparison. The infrared spectral data of these adducts, listed in Table 6, provide the information required to unambiguously exclude the formation of cross-linked resin 20. The mono-adduct $\mathbf{2 1}$ of cyclopentadiene and benzoquinone showed a carbonyl absorption at $1662 \mathrm{~cm}^{-1}$ (entry 1), whereas absorption at $1689 \mathrm{~cm}^{-1}$ is characteristic for the di-adduct 22 (entry 2). Thus, an apparent distinction can be made between the presence of a mono- or a di-adduct, based on infrared spectral data. The immobilized cyclopentadiene adduct 11c only showed a strong absorption at $1663 \mathrm{~cm}^{-1}$ (entry 3), which strongly suggests that this adduct is present only as the mono-adduct. Finally, conclusive information was obtained from the reaction of immobilized cyclopentadiene 5a and the monoadduct 21 of cyclopentadiene and benzoquinone (Scheme 9). The resulting immobilized structure showed a strong carbonyl absorption band at $1688 \mathrm{~cm}^{-1}$, caused by the formation of the di-adduct 23. This proves that the immobilized di-adduct also shows a carbonyl absorption at $1688 \mathrm{~cm}^{-1}$ in the IR-spectrum. The complete absence of this absorbance in immobilized adduct 11c thus excludes the formation of the benzoquinone di-adduct $\mathbf{2 0}$.

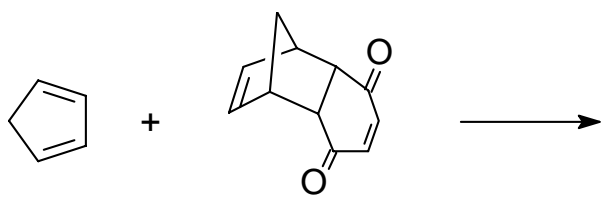

21

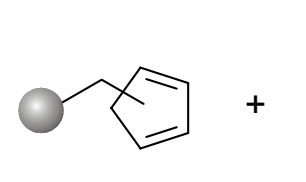

$5 \mathbf{a}$<smiles>O=C1C=CC(=O)C2C3C=CC(C3)C12</smiles>

21<smiles>O=C1C(C2CC3C=CC2C3)C(=O)C2C3C=CC(C3)C12</smiles>

22<smiles></smiles>

23

\section{Scheme 9}


Table 6. Infrared spectral data of benzoquinone adducts

\begin{tabular}{lcc}
\hline Entry & Adduct & Infrared absorptions $\left(\mathrm{cm}^{-1}\right)$ \\
\hline 1 & $\mathbf{2 1}$ & 1662 \\
2 & $\mathbf{2 2}$ & 1689 \\
3 & $\mathbf{1 1 c}$ & 1663 \\
4 & $\mathbf{2 3}$ & 1688 \\
\hline
\end{tabular}

In conclusion, cyclopentadiene was successfully immobilized on the Merrifield resin, affording a monomeric, thermally stable polymeric diene. A loading of up to 1.2 mmole of cyclopentadiene per gram of resin was achieved. The Diels Alder reactivity of the immobilized cyclopentadiene turned out to be reasonably good and comparable with that in solution. The retro-Diels Alder reaction, however, is not generally feasible; it is highly dependent on the nature of the cycloadduct. Chemical modification of the immobilized cycloadducts using methods that generally succeed in solution, were not always successful. Interestingly, the immobilized benzoquinone adduct 11c, for example, appeared totally inert to reactions that proceed in high yields for the non-immobilized benzoquinone adduct 21. The reason for this remarkable inreactivity of the enone moiety in 11c is unknown

As a final conclusion, the Diels Alder/retro-Diels Alder concept is not generally feasible for immobilized cyclopentadiene on polystyrene. The temperatures required to accomplish the cycloreversion of the immobilized cyclopentadiene adducts $\mathbf{1 1}$ is generally too high, both under dynamic and static conditions, to be of practical use. Thus, immobilized cyclopentadiene 5a and its adducts cannot be applied in a combinatorial approach towards pharmaceutically interesting intermediates.

\section{Experimental Section}

General Procedures. Melting points were determined using a Reichert thermopan microscope (uncorrected), or using a Büchi Melting Point B-545 (corrected). $100 \mathrm{MHz}{ }^{1} \mathrm{H}-\mathrm{NMR}$ spectra were recorded on a Bruker AC-100 spectrometer, $200 \mathrm{MHz}{ }^{1} \mathrm{H}-\mathrm{NMR}$ spectra were recorded on a Bruker AC-200 spectrometer and $300 \mathrm{MHz}{ }^{1} \mathrm{H}-\mathrm{NMR}$ and all ${ }^{13} \mathrm{C}-\mathrm{NMR}$ spectra were recorded on a Bruker AC-300 spectrometer using tetramethylsilane (TMS) as an internal standard. FTIR spectra were recorded on an ATI Mattson - Genesis Series FTIR spectrophotometer. DRIFT spectra were recorded using a BioRad FTS-25 spectrometer equipped with an in situ Diffuse Reflectance Infrared Fourier Transform (DRIFT) accessory. For high-resolution mass spectra a double focussing VG7070E mass spectrometer was used. GC/MS was performed on a Varian Saturn GC/MS (Ion trap) equipped with a DB-5 column (30m x 0,25mm). Elemental analyses were performed using a Carlo Erba Instruments CHNS-O EA 1108 element analyzer. Gas chromatographic (GC) analyses were performed on a Hewlett-Packard HP5890II gas chromatograph (flame ionisation detector, FID) equipped with an HP-3396II integrator, using a 
capillary column (HP-1, $25 \mathrm{~m} \times 0.31 \mathrm{~mm} \times 0.17 \mu \mathrm{m})$ and nitrogen at $2 \mathrm{ml} / \mathrm{min}(0.5 \mathrm{~atm})$ as the carrier gas. Diethyl ether was distilled from sodium hydride. Tetrahydrofuran was distilled from sodium and benzophenone. Heptane and dichloromethane were distilled from calcium hydride. Ethyl acetate was distilled from potassium carbonate. Other solvents were distilled if necessary. The Merrifield resin used was obtained from Fluka and cross-linked with 1\% divinylbenzene and contained $1.7 \mathrm{mmole} \mathrm{Cl} / \mathrm{g}$ resin.

\section{Immobilized cyclopentadiene (5a)}

A solution of sodium cyclopentadienylide was prepared according to Hafner and Kaiser. ${ }^{10 a}{ }^{22}$ Sodium (4.93 g, 0.21 mole) was dispersed in refluxing toluene under vigorous stirring. After cooling, toluene was substituted by THF (125 ml, freshly distilled). Under a gentle nitrogen flow, freshly distilled cyclopentadiene $(23 \mathrm{ml}, 0.27 \mathrm{~mol}$ ) was added in portions of $5 \mathrm{ml}$ over a period of $4 \mathrm{~h}$. The pink solution was stirred overnight at room temperature; $15 \mathrm{ml}$ of the resulting solution was added to a suspension of Merrifield resin (5.164 g) in THF (50 ml) under a nitrogen atmosphere. The reaction mixture was stirred gently at $45^{\circ} \mathrm{C}$ for 2.5 hours. The resin was then filtered off and succesively washed with water $(175 \mathrm{ml})$, dichloromethane and methanol. The resin was finally dried under vacuum, yielding immobilized cyclopentadiene 5a. Titration of released $\mathrm{Cl}^{-}$revealed that $1.19 \mathrm{mmole} \mathrm{Cl} / \mathrm{g}$ of the Merrifield resin was functionalised (70\%), giving a loading of 1.15 mmole cyclopentadiene/g resin. IR $(\mathrm{KBr}) \vee\left(\mathrm{cm}^{-1}\right)$ 3062, 3025, 2912, 2842, 1600, 1510, 1492, 1451, 1362, 1018, 898, 755, 691.

\section{General procedure for immobilized Diels Alder adducts (11a-h)}

Immobilized cyclopentadiene 5a (100 mg) was suspended in DMF (10 ml). A large excess ( 10 equiv.) of the dienophile was added and the reaction mixture was stirred at room temperature for 2 to $4 \mathrm{~h}$. The resin was filtered and washed succesively with methanol and dichloromethane (both 3x), and dried in vacuum, affording immobilized Diels Alder adducts 11a-h in yields as listed in Table 2.

Immobilized 4-oxatricyclo[5.2.1.0 $\left.{ }^{2,6}\right]$ dec-8-ene-3,5-dione (11a). IR (KBr) v ( $\left.\mathrm{cm}^{-1}\right)$ 3020, 2918, 2842, 1858 (C=O), 1771 (C=O), 1600, 1492, 1451, 1223, 1077 (C-O), 900, 754, 688.

Immobilized bicyclo[2.2.1] hept-5-ene-2,2,3,3-tetracarbonitrile (11b). IR (KBr) $v\left(\mathrm{~cm}^{-1}\right)$ 3024, 2914, 2250 (CN), 1600, 1491, 1432, 1017, 758, 692. Analysis found: N $6.0 \pm 0.4 \%$, corresponding to $1.25 \pm 0.1 \mathrm{mmole} / \mathrm{g}$ cyclopentadiene reacted.

Immobilized tricyclo[6.2.1.0 $\left.{ }^{2,7}\right]$ undeca-4,9-diene-3,6-dione (11c). IR (KBr) $v\left(\mathrm{~cm}^{-1}\right) 3025$, 2918, 1669 (C=O), 1599, 1486, 1449, 754, 685.

Immobilized diethyl bicyclo[2.2.1]hept-5-ene-2,3-dicarboxylate (11d). IR ( $\mathrm{KBr}) v\left(\mathrm{~cm}^{-1}\right)$ 3024, 2918, 1728 (C=O), 1600, 1492, 1451, 1368, 1027, 754, 691.

Immobilized bicyclo[2.2.1]hept-5-ene-2-carbaldehyde (11e). IR (KBr) v ( $\left.\mathrm{cm}^{-1}\right)$ 3024, 2945, 2895, 2826, 1717 (C=O), 1600, 1507, 1491, 1174, 747, 689. 
Immobilized 4-azatricyclo[5.2.1.0 ${ }^{2,6}$ ]dec-8-ene-3,5-dione (11f). IR (KBr) $v\left(\mathrm{~cm}^{-1}\right)$ 3058, 3020, 2908, 2845, $1768(\mathrm{C}=\mathrm{O}), 1709(\mathrm{C}=\mathrm{O}), 1598,1490,1448,1336,1153,1018,751,688$. Analysis found: N $0.85 \%$, corresponding to 0.64 mmole/g cyclopentadiene reacted.

Immobilized 4-chlorotricyclo[6.2.1.0 $\left.{ }^{2,7}\right]$ undeca-4,9-diene-3,6-dione (11g). IR (KBr) $v\left(\mathrm{~cm}^{-1}\right)$ 3084, 3061, 3027, 2933, 1695 (C=O), 1662, 1599, 1510, 1492, 1451, 1249, 1179, 1026, 747, 686.

Immobilized 4-phenethyl-4-azatricyclo[5.2.1.0 $\left.{ }^{2,6}\right]$ dec-8-ene-3,5-dione (11h). IR (KBr) $v\left(\mathrm{~cm}^{-1}\right)$ 3020, 2909, 2848, 1770 (C=O), 1690, 1680, 1598, 1490, 1448, 1349, 1151, 1025, 747, 686. Analysis found: N 1.12\%, corresponding to 0.95 mmole/g cyclopentadiene reacted.

General procedure for cycloreversion of immobilized adducts (11) under dynamic thermal conditions

Immobilized adducts $\mathbf{1 1}$ were placed in a quartz tube and heated under vacuum (0.05 mbar) using a thermolysis oven. Thermolyses were typically carried out for $2 \mathrm{~h}$ at temperatures between $150-250^{\circ} \mathrm{C}$. The volatile products were trapped in a receiving cooler at $-78^{\circ} \mathrm{C}$. After the thermolysis the receiving cooler was rinsed with dichloromethane (3x). Evaporation of the solvent at reduced pressure gave the cycloreversion products which were analyzed by GC, EI/GC-MS or NMR (Table 3).

Cycloreversion of immobilized adduct (11a). Resin 11a (120 mg; $0.74 \mathrm{mmole}$ of adduct/g resin) was heated at $200^{\circ} \mathrm{C}$ for $3 \mathrm{~h}$, followed by $3 \mathrm{~h}$ at $250^{\circ} \mathrm{C}$, yielding maleic anhydride (7.8 mg; 0.08 mmole $=0.66 \mathrm{mmole} / \mathrm{g}$ ) in $89 \%$ yield.

Cycloreversion of immobilized adduct (11c). Resin 11c (297 mg; $0.71 \mathrm{mmole}$ of adduct/g resin) was heated at $200^{\circ} \mathrm{C}$ for $1 \mathrm{~h}$, affording benzoquinone $(21 \mathrm{mg} ; 0.19 \mathrm{mmole}=$ $0.65 \mathrm{mmole} / \mathrm{g}$ ) in $93 \%$ yield.

Cycloreversion of immobilized adduct (11d). Resin 11d (144 mg; $0.37 \mathrm{mmole}$ of adduct/g resin) was heated at $200^{\circ} \mathrm{C}$ for $1.5 \mathrm{~h}$, affording diethyl maleate $(3.9 \mathrm{mg} ; 0.023 \mathrm{mmole}=$ $0.16 \mathrm{mmole} / \mathrm{g})$ in 43\% yield. EI/GC-MS: m/e (\%) $173\left(\mathrm{M}+1^{+}\right), 127\left(100, \mathrm{M}^{+}-\mathrm{C}_{2} \mathrm{H}_{5} \mathrm{O}\right), 99\left(\mathrm{M}^{+}\right.$$\left.\mathrm{C}_{3} \mathrm{H}_{5} \mathrm{O}_{2}\right), 82\left(\mathrm{M}^{+}-\mathrm{C}_{2} \mathrm{H}_{5} \mathrm{O}-\mathrm{C}_{2} \mathrm{H}_{5} \mathrm{O}\right)$.

Cycloreversion of immobilized adduct (11g). Resin $11 \mathrm{~g}$ (106 mg; $0.90 \mathrm{mmole}$ of adduct/g resin) was heated at $200^{\circ} \mathrm{C}$ for $3 \mathrm{~h}$, affording 2-chlorobenzoquinone (3.3 mg; $0.023 \mathrm{mmole}=$ $0.22 \mathrm{mmole} / \mathrm{g}$ ) in $21 \%$ yield.

\section{Cycloreversion of immobilized adduct (11h)}

a) Resin 11h (238 mg; 0.80 mmole of adduct/g resin) was heated at $200^{\circ} \mathrm{C}$ for $3 \mathrm{~h}$, affording 1phenethyl-2,5-dihydro- $1 H$-2,5-pyrroledione (1.1 mg; $5.5 \times 10^{-3} \mathrm{mmole}=0.02 \mathrm{mmole} / \mathrm{g}$ ) in $3 \%$ yield. ${ }^{1} \mathrm{H}-\mathrm{NMR}\left(200 \mathrm{MHz}, \mathrm{CDCl}_{3}\right) \delta$ (ppm) 7.21-7.40 (m, 5H, arom), 6.66 (s, 2H, HC=CH), 3.76 (t, $J=3.9 \mathrm{~Hz}, 2 \mathrm{H}, \mathrm{NCH}_{2}$ ), 2.89 (t, $J=3.6 \mathrm{~Hz}, \mathrm{CH}_{2} \mathrm{Ph}$ ).

b) Resin $11 \mathbf{h}$ (220 mg; 0.80 mmole of adduct/g resin) was heated at $250^{\circ} \mathrm{C}$ for $2 \mathrm{~h}$, affording 1 phenethyl-2,5-dihydro-1H-2,5-pyrroledione (3.7 mg; 0.018 mmole $=0.08 \mathrm{mmole} / \mathrm{g}$ ) in $10 \%$ yield. ${ }^{1} \mathrm{H}-\mathrm{NMR}\left(200 \mathrm{MHz}, \mathrm{CDCl}_{3}\right) \delta$ (ppm) 7.21-7.40 (m, 5H, arom), 6.66 (s, 2H, HC=CH), 3.76 (t, $J=3.9 \mathrm{~Hz}, 2 \mathrm{H}, \mathrm{NCH}_{2}$ ), 2.89 (t, $J=3.6 \mathrm{~Hz}, \mathrm{CH}_{2} \mathrm{Ph}$ ). 
Chlorinated Wang-resin (15). Wang resin (approx. 2 g, $0.45 \mathrm{mmole} / \mathrm{g}$ ) was suspended in dichloromethane (40 ml). Dichlorotriphenylphosphorane (1.81 g, 3 equiv.) and imidazole ( $0.37 \mathrm{~g}$, 3 equiv.) were added and the mixture was stirred for $17 \mathrm{~h}$. The resin was filtered and washed succesively with dichloromethane and methanol and then dried in vacuo, yielding chlorinated Wang resin 15.

IR $(\mathrm{KBr}) \vee\left(\mathrm{cm}^{-1}\right)$ 3431, 3059, 3024, 2911, 2847, 1600, 1583, 1511, 1492, 1451, 1371, 1302 , 1233, 1173, 1012, 905.

Wang resin-bound cyclopentadiene (16). This resin was prepared according to the procedure described for immobilized cyclopentadiene 5, starting from chlorinated Wang resin 15 (1.87 g). Usual work-up afforded resin 16 (1.99 g).

Wang resin-bound tricyclo[6.2.1.0 $\left.{ }^{2,7}\right]$ undeca-4,9-diene-3,6-dione (17). Resin 16 (501 mg) was suspended in dichloromethane (20 ml). A large excess of $p$-benzoquinone (161 mg; $1.5 \mathrm{mmole}$ ) was added and the reaction mixture was allowed to stir at room temperature for 17 hours. The resin was filtered and washed succesively with methanol and dichloromethane (both 3x), and dried in vacuum, affording functionalised Wang resin 17 (545 mg). Yield: 0.75 mmole cycloadduct/g resin. IR (KBr) $v\left(\mathrm{~cm}^{-1}\right) 1668$.

Cleavage from Wang resin (17). Resin 17 (117 mg) was stirred in a 1:1 mixture of dichloromethane and trifluoroacetic acid/water (95:5) for $2.5 \mathrm{~h}$. The resin was filtered and washed with methanol and dichloromethane. The filtrate was concentrated in vacuo, affording a mixture containing three products in a ratio 2:1:1, which were identified as three isomers of 18.

EI/GC-MS: m/e (\%) (I) 280 (M+, 100), 265 (10.12), 173 (36.48), 160 (19.00), 131 (19.44), 107 (35.07); (II) 280, 252, 173, 158, 107, 91, 77; (III) 280, 174, 158, 107, 71.

\section{Attempted syntheses of immobilized exo-4,5-epoxy-endo-tricyclo[6.2.1.0 $\left.{ }^{2,7}\right]$ undeca-9-en-3,6-} dione (19)

Procedure 1. resin 11c (203 mg) was suspended in acetone (20 ml). A solution of sodium carbonate $(0.5 \mathrm{~g})$ in water $(3 \mathrm{ml})$ was added and the reaction mixture was cooled to $0{ }^{\circ} \mathrm{C}$. A $30 \%$ hydrogen peroxide solution (5 ml) was added and stirred for $1 \mathrm{~h}$, followed by additional stirring at room temperature for $17 \mathrm{~h}$. After addition of water, the resin was filtered off, washed succesively with water, dichloromethane and methanol. According to the IR-spectrum this resin was identical to the starting material.

Procedure 2. resin 11c was suspended in dichloromethane $(4 \mathrm{ml})$ and methanol (2 ml). An aqueous $0.088 \mathrm{M}$ sodium hydroxide solution $(2 \mathrm{ml})$ was added and the reaction mixture was cooled to $0^{\circ} \mathrm{C}$. A $30 \%$ hydrogen peroxide solution $(0.5 \mathrm{ml})$ was added, followed by additional stirring at room temperature for $3 \mathrm{~h}$. After addition of water, the resin was filtered off, washed succesively with water, dichloromethane and methanol. According to the IR-spectrum this resin was identical to the starting material.

Procedure 3. resin 11c (203 $\mathrm{mg}$ ) was suspended in dichloromethane $(15 \mathrm{ml})$. A solution of sodium carbonate $(0.5 \mathrm{~g})$ in water $(4 \mathrm{ml})$ was added and the reaction mixture was cooled to $0^{\circ} \mathrm{C}$. A 30\% hydrogen peroxide solution $(5 \mathrm{ml})$ was added and stirred for $1 \mathrm{~h}$, followed by additional 
stirring at room temperature for $17 \mathrm{~h}$. After addition of water, the resin was filtered off, washed succesively with water, dichloromethane and methanol. According to the IR-spectrum this resin was identical to the starting material.

Procedure 4. resin 11c (232 mg) was suspended in dichloromethane (6 ml). Trichloroacetonitrile (2.0 g; 14 mmole), potassiumhydrogenphosphate $(0.51 \mathrm{~g})$ and $30 \%$ hydrogen peroxide solution $(1 \mathrm{ml})$ was added. The mixture was heated at reflux for $3 \mathrm{~h}$, followed by filtration. The obtained resin was washed succesively with methanol, dichloromethane and diethyl ether and finally dried in vacuo. According to the IR-spectrum this resin was identical to the starting material.

Pentacyclo[10.2.1.1 ${ }^{\mathbf{5 , 8}} \cdot \mathbf{0}^{\mathbf{2 , 1 1}} \cdot \mathbf{0}^{\mathbf{4 , 9}}$ ]hexadeca-6,13-diene-3,10-dione(22).Tricyclo[6.2.1.0 $\left.0^{2,7}\right]$ undeca4,9-diene-3,6-dione 21 (351 mg; 2 mmole) was dissolved in dichloromethane (10 ml). Cyclopentadiene $(0.2 \mathrm{ml})$ was added and the reaction mixture was stirred for $17 \mathrm{~h}$ at room temperature. Dichloromethane was then evaporated, yielding di-adduct 22 as a white powder (380 mg).

${ }^{1} \mathrm{H}-\mathrm{NMR}\left(100 \mathrm{MHz}, \mathrm{CDCl}_{3}\right) \delta$ (ppm) 6.19 (t, 2H), 3.36 (m, 2H), 2.87 (m, 2H), 1.48 (m, 2H). IR $(\mathrm{KBr}) \vee\left(\mathrm{cm}^{-1}\right) 1689(\mathrm{C}=\mathrm{O}), 1448(\mathrm{C}-\mathrm{H}$ stretch cyclohexane ring).

Immobilized pentacyclo[10.2.1.1 $\left.{ }^{5,8} \cdot 0^{2,11} \cdot 0^{4,9}\right]$ hexadeca-6,13-diene-3,10-dione (23). Immobilized cyclopentadiene 5a (351,4 mg) was suspended in dichloromethane (12 ml). A large excess of tricyclo[6.2.1.0 $0^{2,7}$ ]undeca-4,9-diene-3,6-dione 21 (269 mg) was added and the reaction mixture was allowed to stir at room temperature for 5 days. The resin was filtered and washed alternately with methanol and dichloromethane (both 3x), and dried in vacuum, affording the immobilized di-adducts 23 in a yield of $388.0 \mathrm{mg}$, corresponding to a loading of 0.54 mmole of di-adduct/ $\mathrm{g}$ of resin.

IR $(\mathrm{KBr}) \vee\left(\mathrm{cm}^{-1}\right) 1688(\mathrm{C}=\mathrm{O})$.

\section{Acknowledgements}

These investigations were supported by the Netherlands Research Council for Chemical Sciences (CW) with financial aid from the Netherlands Technology Foundation (STW).

\section{References}

1. (a) Ducos, P.; Rouessac, F. Tetrahedron 1973, 29, 3233. (b) Wiel, J.B.; Rouessac, F. J. Chem. Soc., Chem. Commun. 1976, 446. (c) Boland, W.; Jaenicke, L. J. Org. Chem. 1979, 44, 4817. (d) Wiel, J.B.; Rouessac, F. Bull. Soc. Chim. Fr. II 1979, 273. (e) Bloch, R.; Gilbert, L. Tetrahedron Lett. 1987, 28, 423. (f) Takano, S.; Moriya, M.; Ogasawara, K.; J. Org. Chem. 1991, 56, 5982. (g) Takano, S.; Sato, T.; Inomata, K.; Ogasawara, K. J. Chem. Soc., Chem. Commun. 1991, 462. 
2. For reviews see: (a) Lasne, M.-C.; Ripoll, J.L. Synthesis 1985, 121. (b) Ichihara, A. Synthesis 1987, 207. (c) Rickborn, B. In Organic Reactions; Organic Reactions Paquette, L.A., Ed.; Wiley: New York, 1998; Vol. 52, pp 1-223. (d) Klunder, A.J.H.; Zhu, J.; Zwanenburg, B. Chem. Rev. 1999, 99, 1163.

3. (a) Klunder, A.J.H.; Bos, W.; Zwanenburg, B. Tetrahedron Lett. 1981, 22, 4557. (b) Verlaak, J.M.J.; Klunder, A.J.H.; Zwanenburg, B. Tetrahedron Lett. 1982, 23, 5463. (c) Klunder, A.J.H.; Huizinga, W.B.; Sessink, P.J.M.; Zwanenburg, B. Tetrahedron Lett. 1987, 28, 357. (d) Klunder, A.J.H.; Houwen-Claassen, A.A.M.; Kooy, M.G.; Zwanenburg, B. Tetrahedron Lett. 1987, 28, 1329. (e) Lange, J.H.M.; Klunder, A.J.H.; Zwanenburg, B. Tetrahedron Lett. 1989, 30, 127. (f) Houwen-Claassen, A.A.M.; Klunder, A.J.H.; Zwanenburg, B. Tetrahedron 1989, 45, 7134. (g) Lange, J.H.M.; Klunder, A.J.H.; Zwanenburg, B. Tetrahedron 1991, 47, 1509. (h) Zhu, J.; Klunder, A.J.H.; Zwanenburg, B. Tetrahedron 1994, 50, 10597.

4. (a) Hedaya, E.; McNeil, D. J. Am. Chem. Soc. 1967, 89, 4213. (b) Seybold, G. Angew. Chem. 1977, 89, 377. (c) Wiersum, U.E. Recl. Trav. Chim. Pays-Bas 1982, 101, 365. (d) Karpf, M. Angew. Chem., Int. Ed. Engl. 1986, 25, 414. (e) McNab, H. Contemp. Org. Synth. 1996, 3, 373.

5. (a) Klunder, A.J.H.; Zwanenburg, B. In Gas-Phase Reactions in Organic Synthesis; Vallée, Y., Ed.; Gordon and Breach Science Publishers: Amsterdam, 1997; Chapter 2. (b) Brown, R.F.C. In Pyrolytic Methods in Organic Chemistry; Organic Chemistry Monographs, Academic Press: New York, 1980; Vol. 41, pp 259-277.

6. Miura, M.; Akutsu, F.; Usui, T.; Ikebukuro, Y.; Nagakubo, K. Makromol. Chem. 1985, 186, 473.

7. Guhr, K.I.; Greaves, M.D.; Rotello, V.M. J. Am. Chem. Soc. 1994, 116, 5997.

8. Wanner, M.J.; Koomen, G.-J. J. Chem. Soc., Perkin Trans. 1 2001, 1908.

9. Nie, B.; Rotello, V.M. Electrochem. Soc. Proc. 1996, 96, 1212.

10. (a) Hafner, K.; Kaiser, H. Organic Synthesis 1964, 44, 94. (b) Wilkinson, G. Organic Syntheses 1956, 36, 31.

11. Jeffery, G.H.; Bassett, J.; Mendham, J.; Denney, R.C. In Vogel's Textbook of Quantitative Chemical Analysis, $5^{\text {th }}$ Edn, Longman Scientific \& Technical: Burnt Mill, England, 1989; p 355.

12. Bing Yan; Jewel, Jr., C.F.; Myers, S.W. Tetrahedron 1998, 54, 11755.

13. Garner, P.; Ho, W.B.; Grandhee, S.K.; Young, W.J.; Kennedy, V.O. J. Org. Chem. 1991, 56, 5893.

14. Brandsma, L. Preparative Polar Organometallic Chemistry 2, Springer Verlag, Germany, 1990; pp 47-49.

15. Corey, E.J.; Koelliker, U.; Neuffer, J. J. Am. Chem. Soc. 1971, 93, 1489.

16. Singh, P.; Rausch, M.D.; Bitterwolf, T.E. J. Organomet. Chem. 1988, 352, 273.

17. Kresze, G.; Schulz, G.; Walz, H. Liebigs Ann. Chem. 1963, 666, 45.

18. McOmie, J.F.W.; Watts, M.L.; West, D.E. Tetrahedron 1968, 24, 2289. 
19. Veerman, J.J.N. personal communication.

20. Herz, W.; Iyer, V.S.; Gopal Nair, M. J. Org. Chem. 1975, 40, 3519.

21. Pernerstorfer, J.; Schuster, M.; Blechert, S. Synthesis 1999, 1, 138.

22. Hafner, K.; Kaiser, H. Liebigs Ann. Chem. 1958, 618, 148. 